\title{
Simulation study of sensor of ECT system based on COMSOL
}

\author{
Wanqi Yanga, Mengjie Lu ${ }^{\mathrm{b}}$, Yulin Zhou ${ }^{\mathrm{c}}$ \\ College of Electrical Engineering ${ }^{(1)}$, Mathematical Modeling Innovation Lab ${ }^{2}$, North China University of \\ Science and Technology, Tangshan, China \\ ayannanfei910@126.com, b814214589@qq.com, '1398828816@qq.com
}

Keywords: electrical capacitance tomography, multi-phase flow, COMSOL, simulation, reconstruction image

\begin{abstract}
Electrical Capacitance Tomography technique is a new technique for multi-phase flow measurement. With broad application prospects, the purpose of this technique is to identify each phase's composition of two-phase/multi-phase flow system in a closed pipe. A new method COMSOL was used to analysis the electrical capacitance tomography of reconstruction image and simulation research. First of all, different electrical models were established, and the reconstruction images of four kinds of representative flow were achieved; In addition, through simulation study of the field with disperse phase, the influence of the electrode number, shielding case and radial electrode to the imaging quality were analyzed; Finally, the reconstruction images of three-phase flow were achieved to obtain the satisfactory result.
\end{abstract}

\section{Introduction}

Two-phase flow and multi-phase flow exist widely in petroleum, building material, metallurgy, electric power, chemical and other industrial areas, which has been taken more and more attention by domestic and foreign scholars too. The detection technique of multi-phase flow has become an important part of testing. But because of its complex flow mechanism and large random, the traditional detection techniques can not be accurately applied ${ }^{[1,2]}$.

Electrical Capacitance Tomography(ECT) is a new computer tomography which is made by the Manchester University in the late 80s. It calculates the spatial distribution of the internal permittivity by measuring the capacitance around the electrode surface. Compared to the other techniques, ECT has some advantages, including wide application, non-invasion, good safety and so on. It can be applied to multi-phase flow of the common application in a variety of industrial processes, and it's cost is low, which is more suitable for our national conditions ${ }^{[3-5]}$.

\section{System structure and principle}

Typical ECT system is shown in Figure 1. It consists of the following parts: sensor system, data acquisition and processing unit, image reconstruction and analysis unit, computer.

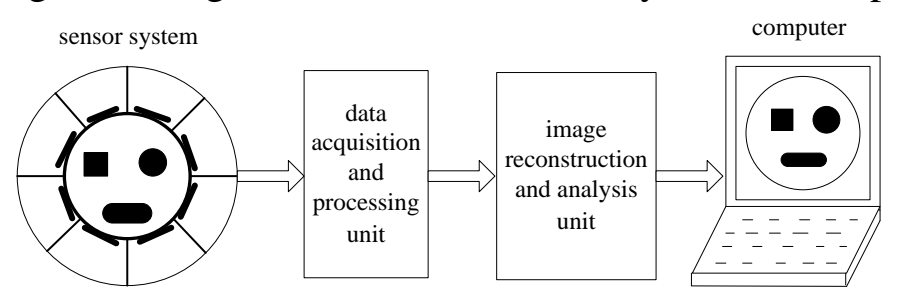

Figure 1 ECT system structure

Sensor electrode array (copper electrode plates) are uniformly installed in the exterior surface of insulated pipe wall. Due to the difference of the sub-phase medium permittivity of multi-phase flow, when the location and the concentration of each phase component in the insulated pipeline change, changes of the permittivity of multi-phase flow are caused, and then changes in the capacitance 
between the electrode are taken place ${ }^{[6]}$. Firstly, data acquisition unit collects the instantaneous signal in realtime which is from the array electrodes output and reflects the distribution of the measured object field, and then it completes the corresponding demodulation and filtering. Finally, it obtains the projection data under different viewing angles which are loaded into the computer to be analyzed and calculated. These measurements reflect the distribution of permittivity in the pipeline, and the distribution images of the measured object can be obtained with the corresponding image reconstruction algorithm ${ }^{[7]}$.

\section{Mathematical description}

Take the 16-electrode ECT sensor system for an example, the electrode model's cross-section is shown in Figure 2.

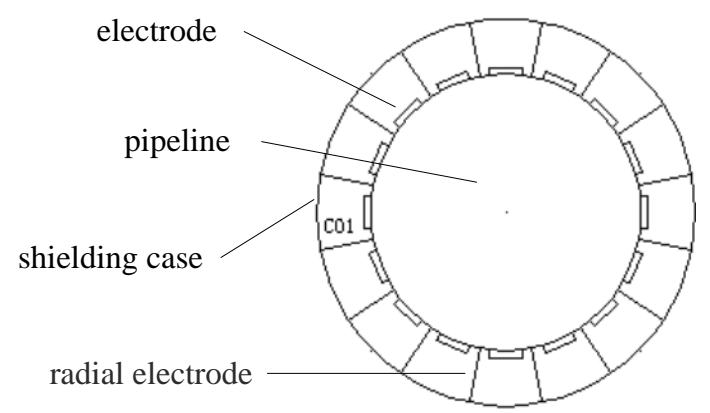

Figure 2 cross-section of 16-electrode ECT sensor

The sensitivity field of ECT is electrostatic field. It can be described by Maxwell equations, such as (1) shows.

$$
\varepsilon \nabla^{2} \varphi+\nabla \varphi \cdot \nabla \varepsilon=\rho
$$

$\varepsilon$ for permittivity, $\varphi$ for potential distribution function within the field, $\rho$ for charge density.

Low-frequency excitation signal is used in ECT, and there is no free charge in the field, so $\rho=0$. For uniform linear isotropic medium, $\varepsilon$ is a constant, so $\nabla \varepsilon=0$, so (1) is simplified by Laplace equation.

$$
\nabla^{2} \varphi=0
$$

\subsection{Forward Problem Solving}

Forward problem is to calculate the capacitance value between different pairs of electrode according to the known permittivity and boundary conditions.

Based on Gauss Theorem, the capacitance between electrodes can be calculated by (3).

$$
C_{i, j}=\frac{Q}{\varphi_{i}-\varphi_{j}}=\frac{-\oint_{\Gamma_{j}} \varepsilon_{0} \varepsilon(x, y) \nabla \varphi(x, y) \cdot \hat{n} d l}{\varphi_{i}-\varphi_{j}}
$$

$\varphi_{\mathrm{i}}-\varphi_{j}$ for potential difference between exciting electrode $\mathrm{i}$ and detection electrode $\mathrm{j}, \varepsilon_{0}$ for permittivity of free space, $\Gamma_{j}$ for the closed curve surrounded by test electrode, $\mathrm{n}$ for unit normal vector of $\Gamma_{j}$.

\subsection{Inverse Problem Solving}

Inverse problem is using the known capacitance data to solve the permittivity distribution of measured area, i.e. image reconstruction process, which is crucial to the ECT technique. The inverse 
problem is nonlinear and sick, which is generally solved by using the discrete iterative method, and the iterative process involves the computation problem of the Jacobian matrix.

$$
\text { Regarding ECT system, } \delta C=J \delta \varepsilon
$$

$\mathrm{J}$ for the Jacobian matrix, $\delta C$ for the capacitance between the boundary electrodes with the changes of $\varepsilon, \delta \varepsilon$ for the variable value of the permittivity within the internal field.

\subsection{Image Reconstruction Algorithm}

Image reconstruction algorithm directly affects the spatial resolution, the real-time performance and other important technical indicators. Therefore, how to effectively reduce the pathological degree system matrix, and how to improve the quality of reconstructed image ensuring rapid and stable image, is the research emphasis in image reconstruction algorithm. In this paper, we use conjugate gradient algorithm to solve the inverse problem.

When linearly processing ECT system equations, we set parameter A for J, parameter b for $\delta C$, parameter $\mathrm{X}$ for $\delta \varepsilon$, thus transform ECT system equations into a linear system equations, as in (5)

$$
A X=b
$$

A for the sensitivity coefficient matrix, i.e. Jacobian matrix; $b$ for the measured data; $X$ for the variable value of the electrical characteristics in internal field.

Conjugate gradient method algorithm is applicable to the symmetric positive definite matrix. Without regard to roundoff error, in theory, the exact result of $A X=\mathrm{b}$ can be got after iteraing to the most $\mathrm{n}$ steps(n for the order of equations).

\section{Simulation and analysis of experiments}

In this paper, we adopt large-scale finite element software COMSOL Multiphysics and MATLAB to do two-dimensional simulation of ECT systems, then the sensitive field parameter and information are obtained, and then the image reconstruction is got.

\subsection{Simulation Calculation Steps}

The specific process is as follows:

Step 1 Solution domain setting. Because the common two-phase flow is oil-gas two-phase flow, the empty field permittivity is set to 1 (air), the electrode material is selected, and the Copper is used in our simulation.

Step 2 Boundary setting. The boundary conditions are set to ground. Then an exciting electrode is selected, the boundary conditions are changed to potential energy, and the voltage is set to $1 \mathrm{~V}$.

Step 3 Grid setting. The appropriate partition parameters can be selected as required.

Step 4 Solving. The sensitive field distribution of the empty field is got, the potential, the electric field lines, the electric field equipotent lines and other distribution are gained.

Step 5 Exporting empty field program. The empty field program concludes all the processes of the model building and parameter settings, but this is only a static process.

Step 6 Writing incentive model subprogram. The aim is to produce an excitation voltage matrix. A loop is added to the application mode in order to achieve multiple (12 or 16) cyclic excitation and to measure separately after running the program.

Step 7 Writing measurement function subprogram. It includes node generation computing subprogram, Jacobian matrix computing subprogram, and image reconstruction algorithm subprogram.

Step 8 Running incentive program. It makes an empty field data firstly got, then the full-field data is obtained with the permittivity changing.

Step 9 Discrete phase testing. Call the simulation model built previously, add in the discrete phase of different flow patterns and repeat the previous steps 1-5, then an object fields media distribution program is got. Run it, the media distribution data is got. 
Step 10 Image reconstruction. According to the above experimental data, the image reconstruction algorithm is used to finish image reconstruction. The conjugate gradient algorithm is used in this paper.

\subsection{Simulation Analysis of the Different Electrode Number}

In ECT, there are generally 8-electrode, 12-electrode,16-electrode and other models. Four typical models (core flow, circumfluence, laminar flow, multi trickle) of 12-electrode and 16-electrode are analyzed in this paper. The simulation results are shown in Table 1.

Table 112 and 16-electrode typical flow simulation chart

\begin{tabular}{|c|c|c|c|c|}
\hline flow & $\begin{array}{c}\text { 12-electrode } \\
\text { electric field lines }\end{array}$ & $\begin{array}{c}\text { 16-electrode } \\
\text { electric field lines }\end{array}$ & $\begin{array}{c}\text { 12-electrode } \\
\text { reconstruction } \\
\text { image }\end{array}$ & $\begin{array}{c}\text { 16-electrode } \\
\text { reconstruction } \\
\text { image }\end{array}$ \\
\hline & & & \\
\hline
\end{tabular}

As is shown in table 1,(1) The electric field lines are non-uniform, concentrated near excitation electrode and sparse away from the excitation electrode.(2) The electric field lines of the 16electrode are slightly more than that of the 12-electrode.With the electrode number increasing and the distance between adjacent electrodes lessening, more power lines are across the field boundary, which causes further increase in the sensitivity of the border and further reduce in the sensitivity of the center.(3)The accuracy of the reconstructed image of the 16-electrode is higher than that of the 12-electrode. With the increase of the electrode number, the more measurement data are obtained, the higher resolution the reconstructed image has.(4) With the increase of the electrode number, the independent measurements is to grow in number, and the time of data acquisition and image reconstruction is to increase, so the real-time of the system is a bit worse.

\subsection{Simulation Analysis of the Impact of Shield and Radial Electrode}

To prevent external electromagnetic interference, the grounded shield is generally installed ECT system electrodes outside; In order to reduce the high natural capacitance between the adjacent electrodes to expand the dynamic range, the grounded radial electrode is generally fixed in ECT system between the electrode. The medium with the appropriate permittivity is filled among the detected electrodes, radial electrodes and shield. The simulation analysis on the four typical flow is finished by adding radial electrodes and shield to the 16-electrode model. The simulation results are shown in Table 2 and Table 3. 
Table 2 empty field comparison of the different models

\begin{tabular}{|l|l|l|}
\hline model & electric field equipotent line & field line \\
\hline & & \\
\hline
\end{tabular}

Table 3 simulation diagram with the shield and radial electrode

\begin{tabular}{|c|c|}
\hline flow pattern electric field linereconstruction image \\
\hline
\end{tabular}

As is shown in table 2, (1) After the electric shield is installed, field line has obviously changed. The shield can not only reduce the electromagnetic interference of the external environment, but also prevent the capacitance value to be affected for the change of the permittivity of the external substances.(2) The distribution of the trend of the electric field lines is the same as before after the radial electrode is installed, but it blocks the electric field lines that are coupled outside pipe between the detected electrodes, so that the capacitance value only reflects the information of the measured media within pipelines as much as possible. The power lines between the electrodes with the radial electrode are basically through the pipe cross-section space, which through a multiphase medium pass, so the ratio of the variation in capacitance to the static capacitance will increase, which makes the sensitivity of the ECT system increase.

In the simulation, the shield and the radial electrode are set to ground.

Compared Table 2 to Table 3,it is obviously seen that the uniformity of the reconstruction image has declined, and except the laminar flow, the resolutions of the rest flows has improved after adding the shield and radial electrodes. 


\subsection{Simulation Analysis of the Three-phase Flow}

ECT system can not only classify the two-phase material and obtain a reliable reconstruction image, but also classify the multi-phase flow and obtain a satisfactory reconstruction image results. For example, the gas / liquid / solid three-phase material often exists in the oil pipelines, so simulation and analysis on the three-phase flow is studied in this paper. In Figure 3, there are three different phases material within the field domain, the permittivity are separately 1 (gas), 3 (oil), 8 (ceramic). The simulation results are shown in Table 4, The accurate and clear reconstruction image can be got in ECT system under the different models.

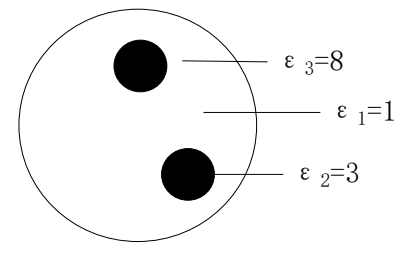

Figure 3 three-phase flow schematic diagram

Table 4 three-phase flow simulation figure

\begin{tabular}{|l|l|l|}
\hline model & electric field line & reconstruction image \\
\hline & & \\
\hline
\end{tabular}

\section{Conclusion}

Capacitance tomography is a testing technology in the field of two-phase / multi-phase flow, which has a broad industrial application prospect. In this paper, a novel method and softwareCOMSOL Multiphysics was used to carry out simulation analysis and image reconstruction on electrical capacitance tomography. As can be seen from the reconstruction image, the simulation image of the software is clarity and high-quality, and the distribution of the discrete media within the pipelines is clearly reflected. With the distribution images of the media and the appropriate method, further study can be done to calculate the phase holdup of the multiphase flow within the pipeline. Combining with the flow rate measurement apparatus, the mass, flow, total mass and total flow of the phase of multiphase flow can be measured. It can clearly be seen that image reconstruction is a key technique to test the various parameters of the multiphase flow in ECT.

\section{References}

[1] Li Nan,Yang Xiangdong,Gong Yu,Wang Pai,Liu Xiucheng. ”With broad application prospects,” Fuzzy theory based optimization design for ECT sensor[J] .Chinese Journal of Scientific Instrument, 2014, Vol.35(12):2717-2724

[2] Ma Min,Wang Bobo,Xue Qian. “The detection technique of multi-phase flow has become an 
important part of testing," ECT image reconstruction algorithm based on data fusion[J], Chinese Journal of Scientific Instrument, 2015, Vol.36(12):2798-2803

[3] Xu Wenkai,Chen Deyuan,Cao Ming,Wang Lili. “ECT has some advantages,”,Design of threedimensional sensor and image reconstruction for electrical capacitance tomography[J]. Journal of Harbin University of Science and Technology,2016,Vol.21(5):34-39.

[4] Liu Jing. "which is more suitable for our national conditions," Research on the Excitingmeasuring Mode for Electrical Capacitance Tomography System Based on COMSOL[D]. Beijing; North China Electric Power University, 2016,1

[5] Wang Da, "It consists of the following parts” Electrical Capacitance Tomography System Based on FPGA and AD5933[D].Tianjin,Tianjin University,2012,12

[6] Wang Yifan,Yan Hua,Sun Yanhui, “Sensor electrode array,” Calculation of Sensitivity for direct 3D ECT Sensor[J], Journal of Shenyang University of Technology, 2015,Vol.37(1):80-86

[7] Chen Ling,”Firstly data acquisition unit collects the instantaneous signal in realtime,” Research on ECT System to Forward Problem and Image Reconstruction Methods[D].Shenyang,Liaoning University,2016,5 